le portiQue $\begin{array}{ll}\text { Le Portique } \\ \text { Revue de philosophie et de sciences humaines }\end{array}$

33 | 2014

Straub !

\title{
À propos de la mort d'Empédocle
}

Jean-Luc Nancy et Jean-Marie Straub

\section{OpenEdition}

\section{Journals}

Édition électronique

URL : http://journals.openedition.org/leportique/2752

DOI : $10.4000 /$ leportique.2752

ISSN : $1777-5280$

\section{Éditeur}

Association "Les Amis du Portique"

Édition imprimée

Date de publication : 1 mai 2014

ISSN : 1283-8594

\section{Référence électronique}

Jean-Luc Nancy et Jean-Marie Straub, «À propos de la mort d'Empédocle », Le Portique [En ligne], 33 |

2014, document 3, mis en ligne le 05 février 2016, consulté le 12 avril 2021. URL : http://

journals.openedition.org/leportique/2752 ; DOI : https://doi.org/10.4000/leportique.2752

Ce document a été généré automatiquement le 12 avril 2021.

Tous droits réservés 


\title{
À propos de la mort d'Empédocle
}

\author{
Jean-Luc Nancy et Jean-Marie Straub
}

1 [Cette retranscription ne gomme pas le style oral du dialogue ni les obscurités - ni, non plus, peut-être, certaines imprécisions - dues à l'improvisation. Les difficultés de ce texte en font le vivant intérêt... et la drôlerie qui n'exclut nullement la profondeur. Il est fait référence au texte de la première version de la tragédie de Friedrich Hölderlin Der Tod des Empedokles et au texte de la troisième version, traduits par Danièle Huillet, et publiés aux éditions Ombres en 1987 et en 1990.]

Benoît Goetz, mai 2011.

Benoît Goetz - Qui est Empédocle?

3 Jean-Luc Nancy - Oui, je veux bien partir de là, c'est une bonne façon de commencer. Qui est Empédocle? C'est une question très difficile, devant ce film, Ce n'est pas une question historiquement difficile. Empédocle était d'abord ce que Jean-Marie Straub a dit tout à l'heure, un Grec d'Agrigente, qui s'est distingué par un comportement particulièrement remarquable à beaucoup d'égards, par son savoir et, comme à l'époque tout était mêlé, par sa réputation de thaumaturge, etc. Nous autres, les philosophes, nous le classons parmi les philosophes. Il fait partie de ceux qu'on appelle les pré-socratiques, bien qu'il soit, comme le faisait remarquer Jean Goetz, contemporain de Socrate.

D'Empédocle, aujourd'hui, nous avons une série de textes, de fragments, qui sont des fragments d'un livre, De la nature, comme les penseurs de cette époque, ceux qu'on appelle d'ailleurs pour cette raison les « physiciens » (ceux qui étudiaient la nature, pas encore les philosophes), en écrivaient. Ils ne s'étaient pas encore donné le nom de philosophes, mais ils étudiaient la nature, ce qui implique, d'une certaine manière, un détournement par rapport à une explication théologique, mythologique du monde. Empédocle, c'est cela. Il est connu pour avoir refusé la royauté. Ce que vous avez entendu dans le film, est attesté historiquement. Les gens d'Agrigente lui ont proposé la royauté, il l'a refusée. D'autre part, il est connu, en dehors de ses œuvres, parce que la légende voudrait que, ou bien il se soit jeté dans l'Etna - en ajoutant ce détail qui apparaît dans une autre version de la tragédie par Hölderlin que ses sandales d'airain 
auraient été rejetées par le volcan - ou bien qu'il ait disparu, sans proprement mourir. Je crois que ce point est très important. Vous l'avez perçu, C'est au centre du film.

5 Premièrement, Empédocle, c'est cela. Deuxièmement, Empédocle, est une figure pour Hölderlin. Le film est fait à partir de la tragédie de Hölderlin. Pour Hölderlin, Empédocle est quelqu'un qui n'a pas d'œuvre, au sens où il ne disposait pas de ces fragments que nous connaissons aujourd'hui... Bien sûr, les récits des historiens anciens, comme Diogène Laërce sur lequel Hölderlin s'est appuyé surtout, rapportent que c'était un penseur, ou un savant, comme vous voudrez. Mais c'est devenu pour Hölderlin, à un certain moment, une figure qui est tout d'abord, je crois... je ne dirais pas tout à fait celle d'un poète, bien que la poésie apparaisse, bien qu'il en soit question dans le texte. Poète, mais surtout à la fois celui qui voit et qui voit là où il n'y a plus à voir, qui voit au-delà du visible, qui voit l'invisible. Et qui voit d'un savoir tel que ce savoir est proprement incommensurable à l'homme. C'est en tout cas cette figure que Hölderlin a cru pouvoir tirer de ce qu'il a connu d'Empédocle. Cette figure, il l'a travaillée, dans le texte que vous avez entendu, mais il a fait trois versions de la tragédie, il s'y est repris à trois fois. D'ailleurs, les Straub ont fait un autre film qui s'appelle Noir péché, qui est fait d'après la $3^{\mathrm{e}}$ version d'Empédocle. Nous avons trois versions de la tragédie, et encore quelques autres fragments et documents, comme un plan développé, dit le plan de Francfort. Plus encore un poème de la même époque, appelé Empédocle. Donc, Empédocle, deuxièmement, c'est Hölderlin.

6 Ce qui ne fait peut-être pas avancer grand-chose. Parce que, qui est Hölderlin? Je ne vais pas vous donner tout le détail biographique, ce n'est pas la question. Je dirais peutêtre que Hölderlin, c'est le premier - je suis presque tenté de dire aussitôt le dernier le premier grand témoin de ce qu'il appelle «le détournement des dieux », le premier grand témoin d'un écart absolu entre l'homme et les dieux, - les dieux n'ayant pour Hölderlin rien de religieux. Vous avez pu voir comment le prêtre, Hermocrate, le prêtre de la cité, est traité dans la tragédie. Mais une incommensurabilité entre l'homme et la totalité ou l'unité, le Un, qui est nommé dans le film à plusieurs reprises «Éther », Le Père Éther (Vater Ether). Mais qui est aussi beaucoup nommé dans la tragédie et le film comme « Nature ». Empédocle est, pour Hölderlin, conformément à beaucoup de choses qu'on peut lire chez lui, le témoin à la fois du rapport de l'homme à l'unité absolue, et de sa séparation. Il est le témoin du déchirement entre l'homme et la Nature, l'Éther, le Un. Mais un témoin extraordinairement complexe et ambigu. Il ne témoigne ni de la pure séparation, ni de la réconciliation. Il est très exactement suspendu entre les deux. C'est du moins ce qu'il me semble. Il me semble, peut-être à tort, que c'est aussi le parti du film. Parce que c'est le parti de Hölderlin. Le film est extraordinairement fidèle au texte de Hölderlin.

7 Troisièmement, Empédocle, c'est qui ? C'est encore... c'est ce que nous avons vu : La Mort d'Empédocle. De cette façon, on pourrait dire, (comme auparavant je disais : c'est Hölderlin) : c'est les Straub, bien sûr. Et ça se donne par quoi ? Ça se donne par un film, ça se donne en tant que film. Il y a dans celui-ci quelque chose qu'il y a ailleurs dans leurs films, mais qui, me semble-t-il, dans celui-ci, est particulièrement sensible, marqué. Toujours ce qui est derrière les personnages, ce qui est toujours là, c'est la nature, à part quelques très exigus éléments de culture - des pieds de colonnes, les marches de la maison d'Empédocle avec les trois esclaves qui sont assis. Autrement, c'est la nature, c'est les arbres... Et il y a au moins deux longs plans, dont un vraiment long - je dirais peut-être au début du dernier tiers du film - où c'est Empédocle qui 
parle, mais où nous ne le voyons pas. C'est un très long plan sur l'Etna au fond, en train d'être découvert par les nuages, et puis les arbres au premier plan. Je pense que le film n'est pas uniquement là, mais qu'il est d'abord énormément là, dans ce choix d'une absence de profondeur de champ, qui fait que la nature dont il est toujours question, la nature qu'il s'agit de retrouver, la nature qu'Empédocle connaît, a connue, retrouvée, vers laquelle il va retourner - et justement, on ne sait pas comment il va y retourner la nature n'est pas distincte. Le film ne nous amuse pas avec une belle nature. Il y a un plan où vous hésitez un petit peu. C'est un plan où il $\mathrm{y}$ a des bouleaux blancs, derrière l'homme de la cabane. Ces bouleaux sont...

Jean-Marie Straub - Ce sont les bouleaux de l'Etna.

9 J.-L. N. - Oui, ce sont les bouleaux de l'Etna. Eux sont nets.

10 J.-M. S. - Je suis ignorant. Empédocle, qui est "botaniste ", nous a expliqué que c'étaient des bouleaux antédiluviens, qui n'existent que sur l'Etna.

11 J.-L. N. - C'est pour ça qu'eux, ils ont droit à un peu plus de netteté que tous les autres arbres qu'on voit.

12 J.-M. S. - Vous êtes dur.

13 J.-L. N. - Non. C'est vous qui êtes dur. Bien sûr, il y a des pins, des branches de pins qui sont au premier plan, qui sont plus nettes, mais à ce moment-là, il y a encore des arrière-plans, qui curieusement sont à plusieurs reprises secoués par un vent beaucoup plus fort que celui qu'on peut percevoir au premier plan dans les ramures des pins.

Dans ce parti-pris, je lis moi plusieurs choses à la fois. À la fois l'absence d'une nature immédiatement réconciliable. Ce ne sont pas les jolies petites fleurs, et les jolis arbres et les feuillages, etc. Cette nature vaut beaucoup plus comme immensité, comme profondeur, comme lointain. Elle vaut aussi comme une sorte d'auréole ou d'aura. À un moment, il y a un plan où Pausanias, le disciple, le jeune disciple, est devant... Tout le fond de l'écran est en feuillage vert pâle, un peu frissonnant. Et justement, Empédocle lui dit une phrase au sujet d'une auréole, d'une aura autour de lui. Je ne me rappelle plus la phrase. Alors, je crois que d'une part, ce détachement des personnages par rapport au fond sert d'abord la parole, en tant qu'il met la parole en avant, que cette parole, il faut l'entendre. Et là, il faut la lire évidemment. Il y a un problème pour ceux qui n'entendent pas l'allemand. Il y a un problème qui est même traité de manière très élégante, très efficace, à certains moments, par la suppression de sous-titres alors qu'il y a des éléments de dialogue qui mériteraient d'être entendus, mais qu'on a choisi de sauter. Évidemment, la condition de spectateur est très différente si on est germanophone ou pas. Mais c'est une autre question dont on pourra reparler.

Comme je ne veux pas faire tout un exposé, développer, je pose simplement ces données, et après, on peut continuer. Je voudrais pour m'arrêter là pour le moment, poser ceci que j'ai déjà annoncé. On ne sait pas ce qui arrive à Empédocle. On ne sait pas. Est-ce qu'il va se jeter dans l'Etna? On a montré l'Etna de façon soulignée. Il est nommé dans le texte une fois. Maintenant, le public n'est pas censé savoir que c'est l'Etna, et on ne nous montre pas un volcan développant un panache, encore moins crachant du feu. Donc, est-il allé se jeter dans l'Etna? A-t-il disparu n'importe où, nulle part? S'est-il évanoui dans la nature? On ne le sait pas et je crois que Hölderlin ne savait pas, ne voulait pas le savoir, ne voulait pas vraiment trancher entre les solutions possibles. C'est peut-être une des raisons pour lesquelles il a fait trois versions. En tout cas, ce qui est remarquable, c'est qu'Empédocle dans le texte écarte très expressément 
la possibilité de la mort. Ou plutôt, il ne l'écarte pas non plus exactement. Il affirme qu'il va mourir. Il affirme qu'il n'y a pas d'autres solutions. Et en plus, il y a un mot du texte qui n'était pas sous-titré, qui est : « la mort des grands est-elle aussi grande? » Il y a une affirmation de la mort, mais cette affirmation de la mort est doublée, non pas d'une négation... Presque ! Il y a une phrase qui affirme qu'il n'y a pas de mort dans la nature. Il répond à Pausanias et il lui dit: "Périr, mais c'est rester gelé, immobile, crois-tu que la nature soit capable de ça, crois-tu que ça arrive à la nature et à la vie ?» La vie ne cesse pas, la nature ne cesse pas. Et en même temps, la manière dont cette unité fondamentale ne cesse pas de se perpétuer à travers tout, cette manière n'est pas exposée, n'est pas réfléchie. Ce qui, pour moi, veut dire beaucoup. Parce que ça veut dire à la fois qu'il n'y a pas de réponse religieuse, qu'Empédocle ne dit pas : « je vais être sauvé ». On peut mentionner au passage que les références christiques plus ou moins visibles ne sont pas très nombreuses, mais sont présentes dans le texte. Les commentateurs les ont relevées à plusieurs reprises. Alors, il m'a semblé qu'il y avait un passage où il dit à Pausanias d'aller préparer un repas et du vin, qui n'est pas gardé dans le dialogue peut-être. C'est un des passages qui a une résonance christique. Il y a quelques résonances christiques, comme chez Hölderlin. Il faut savoir par ailleurs que Hölderlin, c'est à peu près le seul personnage de cette époque, et de peut-être beaucoup d'époques, à avoir tenté de fondre ensemble ou plutôt de tresser ensemble le Christ, Prométhée, Empédocle justement, et d'autres figures. C'est sûr qu'en même temps, il y a quelque chose de l'ordre du sacrifice, d'ailleurs le mot est employé. Mais en même temps, pourtant, ce n'est pas le sacrifice christique, ça n'est pas l'annonce ni la promesse d'un salut, encore moins d'une résurrection ou d'une transfiguration. Je le dis de l'intérieur de la petite boutique philosophique, mais c'est quand même important parce que Hölderlin était camarade de Hegel pendant leurs études. Il connaissait très bien Hegel, et Hölderlin était très philosophe aussi. Il a écrit par exemple sur Kant des choses extrêmement remarquables. Dans La Mort d'Empédocle et nulle part chez Hölderlin, il n'y a la solution hégélienne. La solution hégélienne, c'est quelque chose qui est très connu chez les philosophes, c'est la vie de l'esprit qui se maintient jusque dans la mort, et qui ne passe pas dans la mort. Je crois que c'est important parce que ça donne toute la mesure de la distance entre Hölderlin et la philosophie. Ça permet aussi de bien nommer, en quelque sorte, ce qui est un peu au cœur du film, la vie dont il est question, la vie, la nature, la vie comme union avec le tout de la nature, comme union avec le Père Éther, etc. Cette vie n'est pas une vie plus puissante que la mort. Elle n'est pas non plus une vie qui résiste et qui traverse la mort, comme le veut Hegel - ce sont à peu près les termes de Hegel. Par conséquent, puisqu'on parle beaucoup de résistance à propos du cinéma des Straub, ce qui est intéressant, c'est que, bien sûr, il y a une résistance extraordinaire d'Empédocle, mais cette résistance n'est pas la résistance d'un processus victorieux. C'est plutôt la résistance d'un retrait qui ne cesse pas de se retirer, méprisant de plus en plus le monde des hommes en tant que monde des honneurs, du pouvoir, de la religion, etc. Mais n'affirmant rien d'autre. Je pense que c'est à la fois le très grand mérite d'Empédocle sous les trois noms que j'ai dits. J.-M. S. - Il y a une grande affirmation avant qu'il disparaisse. Il y a deux plans où on voit plus que des paysages. Le premier est le plus long. Il y a une grande affirmation.

17 J.-L. N. - Bien sûr.

18 J.-M. S. - C'est plus qu'Empédocle en personne qui veut disparaitre.

J.-L. N. - Vous avez raison. 

était un Hölderlin jacobin. Hölderlin était jacobin à ce moment-là. Vous avez posé la question avant, vous avez dit : qui est Hölderlin? Ma réponse est très simple. Hölderlin était un jeune poète. Il y a deux Hölderlin. Il y a le Hölderlin d'Empédocle. Les autres versions d'Empédocle, c'est déjà en dehors. Et ensuite, il y a le Hölderlin de la deuxième moitié de sa vie qui était aussi longue que la première. Dont on dit qu'il était fou. Mais enfin, il y a une rupture entre les deux périodes de cette vie-là. Le Hölderlin d'Empédocle... Empédocle, c'est à la fin de la première partie de sa vie, où on sent que tout ça est menacé, et c'est extraordinaire comme un cerveau humain... Et en même temps, on sent que ce cerveau-là travaille tellement bien qu'il est menacé. Ensuite, arrive la deuxième période de sa vie. Le deuxième Empédocle, je ne le connais pas. Ou très mal. Je le connais comme tous les bourgeois qui font semblant de lire. J'ai lu quelques hymnes, les dernières poésies, mais enfin, je ne le connais pas. Tandis que le Hölderlin d'Empédocle, je le connais par cœur. Celui-là exclut même les deux versions ultérieures. C'est un autre... Et qu'est-ce que c'est ? C'est très simple. C'est un jeune poète ambitieux qui rêvait d'écrire une pièce de théâtre pour jouer en plein air après la Révolution en Allemagne. Il voulait être le premier. C'est une sorte de Chénier allemand. Sauf qu'on ne lui a pas coupé la tête. Culture un certain temps. C'était après la Libération. Il avait été un grand résistant et il s'est intéressé à Hölderlin. Pour lui, Hölderlin était un jacobin. Il le démontre. L'affirmation dont je parle, ce paysage, le premier dans le film, le plus long, c'est très simple, c'est plus qu'affirmatif, c'est un appel au communisme. C'est une utopie communiste que Hölderlin développe. Ça commence par «Vous avez soif depuis très longtemps d'inhabituel, et comme d'un corps malade l'esprit d'Agrigente se languit hors de la vieille ornière. Ainsi risque-le! ce que vous avez hérité, ce que vous avez acquis, ce que la bouche de vos pères vous a raconté, enseigné, lois et usages, noms des anciens dieux, oubliez-les audacieusement». Et ça, c'est le développement de ce que moi, personnellement je proclame comme une utopie communiste et rien d'autre! Il ne faut pas avoir peur de le dire.

J.-L. N. - Je vous entends bien. Je vous fais remarquer que j'ai insisté sur ce plan de paysage.

J.-M. S. - C'est tout ce que le communisme a oublié partout où il a fait des tentatives.

27

J.-L. N. - Je suis heureux de vous l'entendre dire, parce que c'est en effet tout ce que le communisme a oublié. Et ça n'est pas non plus une nature conforme à ce que Marx voulait dire quand il disait que l'histoire de l'homme devient l'histoire de la nature. Donc, c'est autre chose. Je veux bien que ce soit le communisme, mais je veux en même temps...

28 J.-M. S. - Je n'ai pas dit que c'était le communisme. C'est un poète qui développe une utopie communiste. 
J.-L. N. - C'est un poète. Sur ce poète, il y a deux autres choses à dire. Ce poète est aussi celui qui avait dit un peu plus tôt, avant Empédocle si je ne me trompe pas : on doit toujours se tenir prêt pour un moment où il faudrait jeter sa plume sous la table et sortir dans la rue pour faire la révolution. C'est Hölderlin qui l'a dit.

J.-M. S. - Je ne le savais même pas.

J.-L. N. - Oui. Mais ça prouve que Hölderlin est comme tous les Allemands de l'époque, il est pris dans le fait que la révolution n'a pas lieu en Allemagne. Et que l'importation de la révolution ensuite par Napoléon, ça sera un peu autre chose.

J.-M. S. - C'est Napoléon qui les a trahis.

J.-L. N. - Là, on est d'accord.

J.-M. S. - Et ce n'est pas les seuls qu'il a trahis.

J.-L. N. - Je veux dire que dans l'Empédocle, l'affirmation de Hölderlin - mais je vais énormément vous choquer - que (pour prolonger ce que je disais avec Hegel), ce n'est pas une affirmation révolutionnaire au sens où ça serait un appel politique à telle ou telle forme d'action politique pour renverser un régime politique. Parce qu'il ne renverse rien.

J.-M. S. - Je ne dis pas que le film a un message qui est un message communiste. Le film est une sorte de biographie d'Empédocle. Et à l'intérieur de ça, il y a ce bloc-là. C'est tout. C'est une biographie extrêmement complexe.

J.-L. N. - En même temps, le poète là-dedans, qui est aussi Empédocle, qui se fictionne lui-même comme Empédocle, c'est celui qui avait écrit - j'ai relevé cette phrase dans un texte en prose de la même période : «le grand poète n'est jamais abandonné de lui-même si loin qu'il s'élève au-dessus de sa propre personne ».

J.-M. S. - C'est de qui ?

J.-L. N. - De Hölderlin.

J.-M. S. - J'aimerais bien avoir ça en allemand. Bien que ce soit de Hölderlin, pour moi, cette phrase-là, c'est du jargon.

J.-L. N. - Non, c'est très clair.

J.-M. S. - Non, c'est de la salade !

J.-L. N. - C'est exactement ce que vous avez dit...

J.-M. S. - Relisez-moi cette phrase, soyez gentil.

J.-L. N. - « Le grand poète n'est jamais abandonné de lui-même si loin qu'il s'élève audessus de sa propre personne ».

J.-M. S. - Il n'y a pas deux mots allemands là-dedans. Je voudrais savoir quel est le traducteur.

J.-L. N. - Je vous enverrai le texte allemand, il n'y a aucun doute, c'est un texte allemand. Les traducteurs de l'édition de la Pléiade n'inventent pas des textes.

J.-M. S. - Les traducteurs en inventent. Vous voulez un bel exemple. Je vous en donne un. Il s'agit de l'italien cette fois. Il y a un monsieur qui disait: "il faudrait des communistes qui ne gâtassent pas le nom " (en italien : che non guastassero il nome). Vous savez comment tous les Français ont eu ce texte? Gallimard, Arnaud, pas n'importe qui : «qui ne fassent pas de tort au Parti »! Alors moi, vous savez, la traduction et la littérature... 
J.-L. N. - Je connais un certain nombre de choses au sujet de la traduction. D'accord, on reviendra sur cette phrase à partir de l'allemand, on échangera là-dessus. En tout cas, pour moi, c'est une des phrases les plus audacieuses de Hölderlin et une des phrases les plus admirables parce que ça veut dire que Hölderlin, exactement dans le sens de ce que vous disiez tout à l'heure, c'est un jeune poète qui est complètement bouillonnant, qui est tendu, tendu en effet par un ensemble dans lequel il y a à la fois la révolution et autre chose, la chose qui est propre à Hölderlin, qui n'est précisément, encore une fois, ni religieuse ni philosophique, ni même politique. Il est capable de dire, en quelque sorte de lui-même, qu'être grand poète, c'est être capable de négliger complètement sa propre personne. Et en ce sens-là, rien ne le menace. Même s'il s'élève. Et qu'est-ce qu'il fait dans (j'allais dire dans Hypérion) dans Empédocle? Il s'élève incroyablement audessus de lui-même, parce qu'il ne faut pas oublier que le nœud de la crise avec les Agrigentins, c'est qu'Empédocle s'est proclamé Dieu. C'est dit, c'est répété, c'est ça qui lui est reproché. C'est un blasphème. Et il semble qu'il y ait quelques attestations historiques pour ça. Je crois, moi, que dans son Empédocle Hölderlin essaie, en prenant les plus grands risques, d'assumer la possibilité de se dire Dieu, tout en étant en même temps en retrait par rapport à elle, tout en la rejetant, en l'écartant. Il y a des moments dans le texte où c'est visible. Mais en comprenant que s'affirmer soi-même comme Dieu, ce n'est plus du tout s'affirmer soi-même. C'est affirmer autre chose. Et c'est s'affirmer autre chose qui porte encore le nom d'Empédocle, mais qui disparaît. Nous sommes entièrement d'accord, qui disparaît dans l'affirmation dont vous dites que la forme la plus forte, c'est ce plan. Mais justement, l'important du film... J.-M. S. - Justement, ce n'est pas lui, ça. Il dit : moi, je m'en vais, faites ça!

51 J.-L. N. - Ce n'est pas lui, mais c'est lui qui parle. C'est sa parole qui devient une parole sans sujet porteur, sans même figure porteuse. J.-M. S. - Il y a aussi un autre aspect. Ce n'est pas seulement le sublime poète, Empédocle. C'est un monsieur qui déclare clairement: je veux me flinguer parce que « je ne veux pas compter les jours, ni être au service du souci et de la maladie ». Il le dit clairement : « Nicht die Tage zählen nicht dienen der sorg und Krankheit ».

J.-L. N. - Oui.

54 J.-M. S. - C'est clair. Ce n'est pas l'idée du sacrifice seulement...

55 J.-L. N. - Il y a le mot sacrifice. Je suis d'accord avec vous, cela pose beaucoup de problèmes. Je ne prétends pas épuiser l'exégèse du texte de Hölderlin. C'est un texte incroyablement touffu, complexe. C'est sûr. Et je suis entièrement d'accord avec vous, c'est-à-dire avec le film, c'est-à-dire avec le fait qu'il y a un très long plan dans lequel on voit seulement le paysage, avec l'Etna au-dessus, et on entend le texte d'Empédocle...

56 J.-M. S. - «Envoyez tout par-dessus bord». Ça commence comme ça. "Vous avez toujours eu soif, vous avez toujours eu soif de quelque chose d'inhabituel, alors allez-y, osez-le, envoyez tout par-dessus bord ». Voilà. Et ensuite, il développe ce que ça veut dire. « Envoyez tout par-dessus bord ».

57 J.-L. N. - Là, nous sommes d'accord. Mais l'important, dans la mesure où vous faites un film, c'est que l'affirmation est donnée comme image. Dans un certain sens, l'image suspend le discours. C'est ça que je trouve très juste, par rapport à Hölderlin, parce que de Hölderlin, nous n'avons ici que du discours, un discours énorme, très long, très complexe, et en partie contradictoire. Mais en même temps, ce que le film saisit, c'est 
justement que ce qui porte ce discours, c'est autre chose qu'une subjectivité, pour prendre ce mot. À moins que ça vous déplaise qu'on...

J.-M. S. - Ça ne me déplaît pas, mais je n'y comprends rien.

J.-L. N. - Allons donc, vous comprenez très bien!

J.-M. S. - L'image suspend le discours, il faut que vous m'expliquiez ce que ça veut dire. Personnellement, je ne vois pas ce que ça veut dire.

J.-L. N. - Je dis que quand vous faites un plan où on entend toujours le discours d'Empédocle, mais où on ne le voit pas, vous faites quelque chose par rapport à l'énonciation de ce discours.

J.-M. S. - Oui, je fais le contraire du Dictateur.

J.-L. N. - Voilà.

J.-M. S. - Le dictateur, on le voit quand il parle.

J.-L. N. - Cela dit, Empédocle, on le voit pas mal quand il parle.

J.-M. S. - Également une utopie... Et ici, je voulais justement, comme Chaplin s'était montré lui-même, je ne voulais pas recommencer. Et comme on avait vu Empédocle à satiété jusque-là, je voulais que les gens respirent.

J.-L. N. - On avait vu Empédocle à satiété ? Ah, dans le film ? Ah oui, je croyais avant le film, parce qu'il n'y en a pas eu d'autres. Oui, très bien. En effet, j'allais vous dire, Empédocle, on le voit. Et il parle. J'en profiterais pour vous poser une question, du coup. Pourquoi Empédocle est-il si jeune dans le film?

J.-M. S. - Parce que c'est un monsieur qui ne veut pas vieillir, c'est clair. Pour moi, le centre du film, c'est un monsieur qui... On aurait dû tous se flinguer à 42 ans, comme Pavese. C'est un peu vache de dire ça, mais enfin... Empédocle lui, s'il en est là, c'est qu'il devrait avoir un peu moins de 42 ans.

J.-L. N. - C'est-à-dire à peu près...

J.-M. S. - Il est presque déjà trop vieux.

1 J.-L. N. - Il est à peu près au milieu de sa vie, comme Hölderlin entre les deux moments dont vous parliez. Oui, j'ai pensé que vous pensiez à Hölderlin. Cela dit, permettez-moi de ne pas être d'accord...

J.-M. S. - Vous avez imaginé un vieillard?

J.-L. N. - Ni vous ni moi ne nous sommes flingués à 42 ans.

J.-M. S. - On a eu tort, c'est tout.

J.-L. N. - Non. Non parce qu'il n'est pas prouvé que nous ayons beaucoup plus que 42 ans.

J.-M. S. - Ça, c'est optimiste !

J.-L. N. - Vous êtes un jeune communiste brûlant d'ardeur.

J.-M. S. - Non, non, je ne suis pas du tout un jeune communiste.

J.-L. N. - Communiste... ce que vous voulez! Vous râlez, vous râlez, vous tapez du poing...

J.-M. S. - Ce n'est pas parce que j'ai parlé d'utopie communiste que je suis un jeune communiste. Non, il ne faut pas me calomnier. 
81 J.-L. N. - Mais je ne vous calomnie pas. Bon Dieu! Mais vous parlez d'utopie communiste, ça veut dire que vous avez 42 ans et non pas 78 . Voilà, c'est tout. L'âge, ça ne veut rien dire.

82

84 a été le sujet, l'objet, non seulement de travail, mais jusqu'à un certain point, d'identification de Philippe Lacoue-Labarthe, mon ami. Et donc, vraiment, je ne vais pas reculer d'un pas en-deçà de Philippe pour aller voir Bertaux Ça m'est égal. Ça m'intéresse beaucoup plus de rencontrer Straub. Maintenant, si ça doit vous faire vraiment plaisir, je veux bien ouvrir le Bertaux, mais je vous garantis que je ne le lirai pas. Bertaux n'est pas Philippe. Bertaux est un monsieur très extérieur, qui a l'avantage d'être extérieur. Il n'a pas une conception et une interprétation de Hölderlin. Il n'est pas un acteur culturel. Les gens qui ont des interprétations sont les acteurs. Ils ne veulent pas produire un texte et en faire cadeau au public, ils veulent l'interpréter. Voilà, Bertaux n'était pas un interprète. le film... le film 
Spectateur - C'est ça. Et des fois, juste après le point, il termine le point, et ensuite, il ne fait pas la pause au point, il fait la pause après le point. Il fait un bout de la phrase suivante, il s'arrête et hop, il reprend après.

J.-M. S. - Mes beaux enfants, il y a rien de mystérieux là-dedans. C'est tout simplement qu'on a respecté les vers. C'est-à-dire qu'on marque les vers. Quand un vers s'arrête, même si le complément arrive sur l'autre vers, il y a en tout cas une césure, et souvent une pause, qui peut être variable de longueur. C'est tout. On respecte les vers. S'il y a un texte qui consiste en vers, il n'y a aucune raison de le transformer en prose journalistique. C'est tout. Par contre, les sous-titres, pour autant que ce soit possible, respectent la structure du parler qui est une structure qui correspond aux vers.

116 J.-L. N. - C'est ce que je voulais dire.

117 Spectateur-Monsieur!

118 J.-L. N. - Je veux compléter la réponse. Parce que je veux dire que ce respect de la métrique de Hölderlin a des raisons aussi chez Hölderlin lui-même. Pas seulement parce que Hölderlin écrit en vers. Mais parce que pour Hölderlin, la métrique, la prosodie a une portée - comment est-ce qu'il faudrait dire, quel mot employer ?ontologique, essentielle. Pour Hölderlin en particulier, chose qui peut surprendre, la pensée est métrique. Ce qui est déjà une manière de saisir le poème, de faire de la poésie, qui signifie que pour penser, la pensée doit passer par la métrique. Le respect de la métrique de Hölderlin est le respect de quelque chose qui est extrêmement important. Dont, si on avait le temps, on pourrait montrer que ça a affaire avec le fond de la pensée de Hölderlin dans Empédocle.

Spectateur - On a beaucoup évoqué la présence de la nature comme sujet dans le texte, dans ce film. Mais en réalité, cette nature, me semble-t-il, c'est une question que je veux poser à l'auteur $d u$ film, Monsieur Straub, cette présence de la nature n'est pas telle seulement dans ce film d'Empédocle, mais dans la lecture de Dante d'hier soir, par exemple, il y avait aussi une pareille présence pour ainsi dire de la nature. Dans Antigone, pareillement, pour ainsi dire, une même présence de la nature. Ma question est la suivante, toute simple : quel sens, quelle signification donne-t-il, Jean-Marie Straub, à la nature dans ses films?

120 J.-M. S. - Ce n'est pas parce que c'est Hölderlin qu'il y a de la nature. Je veux dire, à la limite, je m'en fous de la nature de Hölderlin, je ne sais même pas ce que c'était. Je ne peux que m'en foutre. Comment est-ce que je saurais ce que c'est que la nature de Hölderlin. Par conséquent, on a fait des films où cette chose-là était un élément important, cette chose-là que vous appelez la nature, et ça continue. C'est tout. Ça varie avec chaque film, mais c'est toujours notre nature. Ça ne peut être que la nôtre et pas celle de Hölderlin.

121 J.-L. N. - Hölderlin dit la nature, il ne se l'approprie pas. Monsieur insistait simplement sur la présence de la nature, du paysage filmé. J'ai quand même, quitte à vous déplaire beaucoup, j'ai un petit bout d'interprétation en plus, à partir justement du dernier chant de Dante. Dans le dernier chant de Dante, la nature que vous montrez, que vous faites éclater après un long moment d'écran vide, cette nature vraiment solaire, avec un champ de blé brûlé, avec un instrument aratoire peut-être sur lequel on s'est interrogé... Cette nature est exactement à la place de ce qui arrive à la fin de la Divine comédie. Ce qui arrive à la fin de la Divine comédie, la Somma Luce, ce n'est pas du tout la nature, c'est la Sainte Vierge. 
J.-M. S. - Il y a un texte de Hölderlin qui commence par : «Ô lumière céleste ! ». C'est grâce à ce texte-là que j'ai réussi à me forcer à m'intéresser au texte de Dante. Si on regarde bien, malgré tout le respect que j'ai pour Dante, qui m'a toujours ennuyé excusez-moi, je peux dire ce que je pense, après tout, je suis un plouc du plateau lorrain ! - comparé à l'hymne à la lumière de Hölderlin, le très beau texte de Dante, c'est un peu du pipi de chat.

J.-L. N. - Dont acte. Donc, il y a aussi nature et nature. La nature dans le film n'est pas, elle, du pipi de chat.

124 J.-M. S. - Je ne crache pas sur le film qu'on a fait. C'est quoi la nature ? On a choisi le plein air. On aurait pu tourner ça dans un espace clos. On a choisi le plein air. C'est tout. Il y a deux parties, une partie au bord de la mer à Ragusa, à trois minutes de Donnafugata. D'ailleurs, on voit Donnafugata à un moment dans le premier plan, dans un trou. Et puis, il y a une deuxième partie sur les pentes de l'Etna, c'est tout. C'est un décor théâtral. Il se trouve qu'on le respecte ce décor, en tant que décor. Et donc, on respecte la nature, c'est tout. Tandis que dans l'autre truc, c'est un décor théâtral, c'est tourné dans un endroit qui n'est pas du tout un champ de blé brûlé, c'est un pré qu'ils ont égalisé, ils ont été trop paresseux pour emmener toute la machine agricole qui restait. J'ai tremblé pendant deux ans, je me suis dit: je ne pourrai plus tourner là-bas parce qu'ils m'auront enlevé ça. Et j'arrive là et il restait un quart de la machine. J'étais content, parce que je voulais l'asseoir là-dessus et pas sur une chaise de jardin.

125 J.-L. N. - Mais la nature, je l'ai dit tout à l'heure, c'est assez simple, dans le cas de Hölderlin, parce que dans le cas de Dante, il n'est pas question de nature. La nature dans le texte de Hölderlin n'est pas ce que je sens qui vous irrite un peu quand on parle de nature. Ce n'est pas du tout la nature opposée à la culture. Heidegger aurait dit : ce n'est pas du tout la natura des Latins. C'est plutôt, si on veut commencer par là, la phusis des Grecs. La nature dans La mort d'Empédocle porte plusieurs noms. " Nature » est un nom important, qui revient quand même plusieurs fois.

126 J.-M. S. - Il y a aussi le De Rerum natura d'un certain Lucrèce.

127 J.-L. N. - Ça, justement, c'est tout à fait autre chose. Lucrèce pour qui il y a une nature devant lui, qui tend déjà à devenir objet jusqu'à un certain point...

128 J.-M. S. - Moi, la nature, je m'en fous. Elle m'intéresse parce qu'elle me permet de faire des images érotiques. C'est tout. Ça me permet de faire des images érotiques. La nature, ça ne signifie rien pour moi, la nature. Ce n'est pas du tout ce que vous croyez, que j'ai horreur de la nature ou des choses comme ça. Je m'intéresse à la lumière. Je m'intéresse à l'air. Et je m'intéresse à ce qui bouge. Je suis comme un gosse.

129 J.-L. N. - Et vous filmez comme un gosse un texte dans lequel le mot de nature est employé peut-être vingt fois et dont on entend bien qu'il est équivalent à ceux de "Éther", "Père Éther", et de "vie".

130 J.-M. S. - On en revient à l'utopie communiste.

131 J.-L. N. - Oui.

132 J.-M. S. - L'introduction de l'utopie communiste, qui est très brève, se termine par « et levez, comme des nouveau-nés, les yeux vers la divine Nature ». Je ne crache pas làdessus.

133 J.-L. N. - Personne n'a dit que vous crachez dessus. Simplement, c'est vrai que ça déplace beaucoup l'idée de nature. C'est tout. Et vous suivez très bien Hölderlin pour ça. 

germanophone puisque je ne connais pas la langue allemande, néanmoins, ce film, en le voyant comme ça de manière brute, ce qui m'a surpris, c'est qu'on sent très bien, alors que le texte, vous l'avez dit vous-même M. Nancy, est touffu, et quand on le découvre comme ça sous-titré et qu'on n'a pas le loisir de revenir sur un ouvrage quand on le lit, ça va assez vite et c'est difficile. Mais néanmoins, ce qu'on sent quand même très bien, ce que j'ai senti en tout cas, on a vu un affrontement extrêmement fort, presque insoutenable, dramatique, à un moment donné, sur ce qu'on pourrait aujourd'hui appeler des forces conservatrices et face à cela, quelqu'un qui effectivement voulait, sinon les bousculer, les renverser, mais en tout cas, être en rupture avec ces forces-là. Ça, ça se sent bien sûr par le texte, mais ça se sent aussi dans la façon dont c'est mis en scène, dans la façon dont les acteurs... Il y a une scansion permanente. Le texte est scandé de telle sorte, il est presque chanté par moment. Même si on ne comprend pas l'allemand, on est complètement pris par cette tension qui est créé par les intonations, quelques gestes même par moment, les regards, etc. Et notamment aussi le jeu avec le jeune, son jeune - je ne sais pas comment on pourrait dire - son jeune disciple qui incarne en même temps l'avenir. À la fin, ils reviennent le chercher, mais il dit: les rois, c'est terminé. Il le dit. Je ne sais pas s'il faut l'interpréter vraiment aussi naïvement, mais il le dit. Il dit : le temps des rois, c'est terminé. 
partir, on ne sait pas trop Mais il me semble avoir perçu dans le générique final un grondement. Je ne sais pas si c'est la raison qui tonne en son cratère, mais on entend quand même un grondement.

J.-M. S. - C'est un orage fortuit qu'on a subi avant la fin du tournage. Par contre, ce que je peux vous dire, c'est que le violon un peu gringrin, que vous entendez au début, qui est un extrait d'une partition de Bach pour violon, ça, c'est lui qui joue. Le soir à 6 heures sur la terrasse de l'hôtel, il s'exerçait avec son violon.

\section{AUTEURS}

\section{JEAN-LUC NANCY}

Jean-Luc Nancy est Professeur de philosophie à l'Université de Strasbourg jusqu'en 1994. Ami de Jacques Derrida et Philippe Lacoue-Labarthe, il est un spécialiste reconnu de la grande tradition 
philosophique allemande. Il s'intéresse aussi au cinéma et aux images et a écrit L'Évidence du film sur Abbas Kiarostami et $\mathrm{Au}$ fond des images. 